\title{
Neuer Verhaltenskodex der pharmazeutischen Industrie
}

\author{
T. Witzig
}

Seit dem 1. Januar 2004 gilt der neue Verhaltenskodex der pharmazeutischen Industrie in der Schweiz (Pharmakodex) [1]. Er ersetzt den bisherigen PharmaFachwerbungs-Kodex (PFK). Neben der Fachwerbung für Arzneimittel regelt der Pharmakodex neu auch die Beziehungen der Pharmaunternehmen zu Ärzten und Apothekern, und zwar bei Veranstaltungen zur Fort- und Weiterbildung sowie bei der klinischen Arzneimittelforschung. Ziel ist die Vermeidung von Interessenkonflikten.

\author{
Korrespondenz: \\ Tobias Witzig \\ SGCI Chemie Pharma Schweiz \\ Nordstrasse 15 \\ Postfach \\ CH-8035 Zürich \\ Tel. 013681736 \\ Fax 013681737
}

E-Mail: tobias.witzig@sgci.ch
Der Pharmakodex ist eine ethisch motivierte Vereinbarung der Pharmaunternehmen. Sie dokumentieren damit ihr Bekenntnis zur Eigenverantwortung und Selbstkontrolle in Bereichen, in denen Interessenkonflikte $\mathrm{zu}$ vermeiden sind. Dies gilt namentlich für ihre Beziehungen zur Ärzte- und Apothekerschaft, die sich bei Veranstaltungen zur Fort- und Weiterbildung sowie bei der klinischen Forschung ergeben können.

Interessenkonflikte sollen bei Veranstaltungen zur Fort- und Weiterbildung vermieden werden. Deshalb soll der zeitliche Aufwand für die Vermittlung wissenschaftlicher oder fachlicher Information denjenigen für das Rahmenprogramm (Gastfreundschaft und allfällige Unterhaltung) deutlich überwiegen. Ausserdem sieht der Pharmakodex vor, dass sich die teilnehmenden Fachpersonen grundsätzlich mit einem angemessenen Beitrag an den Kosten der Veranstaltung beteiligen.

Interessenkonflikte sollen ferner vermieden werden, wenn Unternehmen klinische Versuche mit Arzneimitteln unterstützen. Im Interesse der Transparenz ist ein solches Sponsoring immer vertraglich zu regeln. Der Pharmakodex sieht dafür das 6-Augen-Prinzip vor: Neben dem Sponsorunternehmen und dem verantwortlichen Prüfer muss auch die Institution (Universität, Fakultät oder Departement, Klinik, Stiftung, Forschungsorganisation u.a.), bei der ein klinischer Versuch durchgeführt wird, den Vertrag unterzeichnen. Darüber hinaus hat das Sponsorunternehmen darauf zu achten, dass der verantwortliche Prüfer und seine Mitarbeiter den Versuch unabhängig von den Interessen des Unternehmens durchführen und auch kein eigenes finanzielles Interesse an den Versuchsergebnissen haben.
Schliesslich regelt der Pharmakodex weiterhin die Fachwerbung für Arzneimittel. Für diese Art Werbung, die sich ausschliesslich an die Fachleute (Ärzte, Apotheker, Drogisten) richtet, gelten andere Regeln als für die Publikumswerbung. Anders als Laien können Fachleute aufgrund ihrer Ausbildung und beruflichen Erfahrung den Nutzen und die Risiken von Arzneimitteln beurteilen. Eine Täuschung oder gar Gefährdung durch Arzneimittelwerbung ist bei Fachleuten somit in der Regel ausgeschlossen. Aus diesem Grund ist die Fachwerbung aus gesundheitspolizeilicher Sicht auch kaum je eine Gefahr für das Publikum. Die Überwachung der Publikumswerbung ist Sache der Heilmittelbehörde (Swissmedic).

Fachwerbung vergleicht oft Arzneimittel miteinander, was zu Konflikten wegen unlauteren Wettbewerbs führen kann. Um solche zu vermeiden, verlangt der Pharmakodex, dass die Fachwerbung genau, ausgewogen, objektiv und fair zu sein hat, und bestimmt im Detail, wie diese Gebote umzusetzen sind. Dabei berücksichtigt der Pharmakodex auch die entsprechenden Bestimmungen des staatlichen Heilmittelrechts.

Im Sekretariat von SGCI Chemie Pharma Schweiz sorgt ein praktizierender Arzt im Teilzeitmandat dafür, dass die Bestimmungen des Pharmakodexes eingehalten und fehlbare Unternehmen veranlasst werden, Verstösse zu beheben oder in Zukunft zu vermeiden. Allfällige Meinungsverschiedenheiten zwischen den beteiligten Unternehmen werden dabei im Rahmen eines Mediationsverfahrens einvernehmlich beigelegt. Dieses hat innerhalb enger Fristen stattzufinden und soll in der Regel höchstens 25 Arbeitstage dauern. Scheitert es, so steht der Weg zur Behörde (Swissmedic) oder zum Richter (Klagen wegen unlauteren Wettbewerbs) offen, was indessen die Ausnahme bleiben sollte.

Verstösse gegen Art. 33 des Heilmittelgesetzes (HMG) [2] fallen nicht unter den Geltungsbereich des Pharmakodexes. Diese Vorschrift verbietet, die Arzneimittelauswahl und damit den therapeutischen Entscheid der Medizinalperson mit finanziellen Anreizen zu beeinflussen. Zuständig für den Vollzug dieser Vorschrift 
a Schweizerischer Fachverband der Hersteller rezeptfreier Heilmittel, www.assgp.ch.

b Verband der forschenden pharmazeutischen Firmen der Schweiz, www.interpharma.ch.

c Vereinigung der Importeure pharmazeutischer Spezialitäten, www.vips.ch. ist ausschliesslich Swissmedic. Da indessen das Vorbeugen von Interessenkonflikten eines der Hauptziele des Pharmakodexes ist, trägt er indirekt dazu bei, mögliche Verstösse gegen Art. 33 HMG zu vermeiden.

Motiv für den neuen Pharmakodex sind die gestiegenen ethischen Anforderungen an das Verhalten der Unternehmen. Mit dem Pharmakodex verpflichtet sich die Industrie auf Verhaltensregeln, die für die Ärzteschaft analog bereits gelten. So erliess die Schweizerische Akademie für Medizinische Wissenschaften im September 2002 Empfehlungen zur Zusammenarbeit zwischen Ärzteschaft und Industrie [3]. Diese richten sich an die Ärzte. Auch die SAMW-Empfehlungen tragen dazu bei, Interessenkonflikte zu vermeiden, wenn Ärzte an von der Industrie gesponserten Veranstaltungen zur Fort- und Weiterbildung sowie an klinischen Forschungsprojekten teilnehmen. Gleiches gilt für die Leitlinien der FMH vom 21. November 2003 zur Anerkennung von Fortbildungsveranstaltungen [4].
SGCI Chemie Pharma Schweiz verfügt seit 1969 über einen Verhaltenskodex für Pharmaunternehmen. Die schweizerische Heilmittelbehörde (heute Swissmedic) hat die eigenverantwortliche Überwachung namentlich der Fachwerbung durch SGCI Chemie Pharma Schweiz von jeher anerkannt. Sie unterstützt auch den neuen Pharmakodex, der von den Partnerverbänden von SGCI Chemie Pharma Schweiz (ASSGPa, Intergenerika, Interpharma ${ }^{\mathrm{b}}$, VIPS $^{\mathrm{c}}$ ) mitgetragen wird.

\section{Literatur}

1 www.sgci.ch/plugin/template/sgci/*/11386.

2 www.admin.ch/ch/d/sr/812_21/a33.html.

3 SAMW. Zusammenarbeit Ärzteschaft - Industrie. Empfehlungen der Schweizerischen Akademie der Medizinischen Wissenschaften. Schweiz Ärztezeitung 2002;83(41):2165-71. www.samw.ch/ content/Dokumente/d_EmpfAeI.pdf.

4 FMH. Leitlinien der FMH zur Anerkennung von Fortbildungsveranstaltungen im Rahmen der FBO. Schweiz Ärztezeitung 2004;85(1/2):16. www.saez.ch/pdf/2004/2004-02/2004-02-1338.pdf.

\title{
Nouveau code de bonnes pratiques de l'industrie pharmaceutique
}

\author{
T. Witzig
}

Le nouveau code de bonnes pratiques de l'industrie pharmaceutique en Suisse (Code pharmaceutique) [1] est entré en force le 1er janvier 2004. Il remplace le Code de promotion pharmaceutique professionnelle (CPP) en vigueur jusqu'ici. Désormais, outre la publicité pour les médicaments destinée aux professionnels, le Code pharmaceutique réglemente aussi les rapports des entreprises pharmaceutiques avec les médecins et les pharmaciens dans le domaine du soutien à la recherche clinique sur les médicaments ainsi que lors de manifestations visant la formation postgraduée et continue de professionnels. Son but est d'éviter des conflits d'intérêts.

\author{
Correspondance: \\ Tobias Witzig \\ SGCI Chemie Pharma Schweiz \\ Nordstrasse 15 \\ Case postale \\ CH-8035 Zurich \\ Tél. 013681736 \\ Fax 013681737
}

E-mail: tobias.witzig@sgci.ch
Le Code pharmaceutique est une convention à motivation éthique souscrite par les entreprises de l'industrie pharmaceutique, qui manifestent en le signant leur volonté de se responsabiliser et de s'astreindre à un autocontrôle dans des domaines où il convient de prévenir des conflits d'intérêt. A cet égard, il concerne en particulier les relations pouvant exister entre l'industrie d'une part et le corps médical et les pharmaciens d'autre part à l'occasion de manifestations en faveur de la formation postgraduée et continue ou de projets de recherche clinique.

Il s'agit donc d'éviter des conflits d'intérêts lors de manifestations en faveur de la formation postgraduée et continue des professionnels. Pour cette raison, les dépenses de temps consenties à la transmission d'informations scientifiques ou spécialisées doivent l'emporter nettement sur celles qui sont destinées au programme-cadre (hospitalité et divertissements éventuels). En outre, le Code pharmaceutique prévoit que les professionnels participant à une manifestation versent une contribution adéquate aux frais.

Il s'agit aussi d'éviter des conflits d'intérêts dans les cas où des entreprises soutiennent des essais cliniques portant sur des médicaments. Par souci de transparence, ce type de promotion doit toujours être réglementé par contrat. Le Code pharmaceutique prévoit à ce titre «le principe des six yeux»: le contrat doit être signé non seulement par l'entreprise qui promeut l'essai cli- 
nique et la personne responsable de la réalisation de l'essai, mais aussi par l'institution (université, faculté ou département, clinique, fondation, organisation de recherche ou autres) dans ou avec laquelle l'essai clinique est réalisé. De plus, l'entreprise qui promeut un essai clinique doit veiller à ce que l'investigateur responsable et ses collaborateurs effectuent l'essai indépendamment des intérêts de l'entreprise et n'aient aucun intérêt financier dans les résultats de cet essai.

De plus, le Code pharmaceutique réglemente la publicité pour les médicaments destinée aux professionnels. Cette publicité, destinée exclusivement aux médecins, aux pharmaciens et aux droguistes, est soumise à d'autres règles que la publicité visant le grand public. Compte tenu de sa formation et de son expérience professionnelle, le personnel médical est bien davantage en mesure que ne le sont des profanes d'évaluer les risques ou les avantages d'un médicament. Le risque qu'il court d'être induit en erreur ou de voir sa santé mise en danger par des affirmations publicitaires est dès lors quasi nul. Voilà pourquoi, d'un point de vue de police sanitaire, la publicité pour les médicaments destinée aux professionnels ne constitue pas un danger réel pour le grand public. En revanche, la surveillance de la publicité destinée à ce public est l'affaire exclusive de l'autorité officielle suisse (Swissmedic).

La publicité destinée aux professionnels compare souvent les médicaments les uns aux autres, ce qui peut susciter des conflits pour concurrence déloyale. Pour éviter ces conflits, le Code pharmaceutique exige que la publicité destinée aux professionnels soit exacte, pondérée, objective et honnête, et il réglemente en détail l'application de ces prescriptions. Ce faisant, il prend aussi en considération les dispositions de la législation nationale sur les médicaments.

$\mathrm{Au}$ secrétariat de SGCI Chemie Pharma Schweiz, un praticien mandaté à temps partiel par SGCI Chemie Pharma Schweiz veille à ce que les dispositions du Code pharmaceutique soient respectées et que les entreprises ayant commis une infraction prennent les mesures correctives qui s'imposent ou s'engagent à ne plus la commettre à l'avenir. Le secrétariat assure une médiation en vue du règlement à l'amiable d'éventuels différends entre les entreprises concernées. Cette procédure doit être exécutée dans les plus brefs délais et ne peut s'étendre en principe sur plus de 25 jours ouvrables. Si elle échoue, la voie est ouverte pour des dénonciations auprès de l'autorité compétente (Swissmedic) ou du juge (plainte en concurrence déloyale), processus qui devrait toutefois demeurer l'exception.
Les infractions à l'art. 33 de la loi sur les produits thérapeutiques (LPT) [2] ne relèvent pas du domaine d'application du Code pharmaceutique. Cette disposition légale interdit d'influencer le choix des médicaments et les choix thérapeutiques du personnel médical par des incitations financières ou matérielles. Les cas d'inobservation de l'art. 33 sont exclusivement de la compétence de Swissmedic. Néanmoins, puisque la prévention des conflits d'intérêts est l'un des objectifs principaux du Code pharmaceutique, celui-ci contribue indirectement à éviter les transgressions de l'art. 33 LPT.

Le principal facteur qui a motivé la publication du nouveau Code pharmaceutique est le renforcement des exigences d'ordre éthique imposées aux entreprises. En signant ce code, l'industrie s'astreint à des règles de comportement qui ont déjà cours pour le corps médical. C'est ainsi qu'en septembre 2002, l'Académie Suisse des Sciences Médicales a publié des recommandations en matière de collaboration corps médical - industrie [3]. Ces recommandations, qui visent aussi à éviter des conflits d'intérêts, s'appliquent aux médecins participant à des manifestations liées à la formation postgraduée et continue ou à des projets de recherche clinique parrainés par l'industrie. Poursuivent le même but les directives du 21 novembre 2003 de la FMH pour la reconnaissance de sessions de formation continue [4].

SGCI Chemie Pharma Schweiz a institué un code de bonnes pratiques à l'usage des entreprises pharmaceutiques dès 1969. Depuis toujours, l'autorité suisse compétente en matière de médicaments (aujourd'hui Swissmedic) confie en priorité à SGCI Chemie Pharma Schweiz le soin de surveiller la publicité destinée aux professionnels. Elle salue également le nouveau Code pharmaceutique, qui est cosigné par les associations partenaires de SGCI Chemie Pharma Schweiz (ASSGPa, Intergenerika, Interpharma ${ }^{\text {, }}$ et VIPS $\left.^{c}\right)$.

\section{Références}

1 www.sgci.ch/plugin/template/sgci/*/11387.

2 www.admin.ch/ch/f/rs/812_21/a33.html.

3 ASSM. Collaboration corps médical - industrie. Recommandations de l'Académie Suisse des Sciences Médicales. Bull Med Suisses 2002;83(41): 2172-8. ww.samw.ch/content/Dokumente/ f_EmpfAeI.pdf

4 FMH. Directives de la FMH pour la reconnaissance de sessions de formation continue dans le cadre de la RFC. Bull Med Suisses 2004; 85(1/2):16. www.saez.ch/pdf/2004/2004-02/ 2004-02-1338.pdf. 